\title{
ON TWO DAMAGE ACCUMULATION MODELS AND THEIR SIZE EFFECTS
}

\author{
F. BALLANI,*** \\ D. STOYAN* AND \\ S. WOLF, ${ }^{*}$ TU Bergakademie Freiberg
}

\begin{abstract}
Two cumulative damage models are considered, the inverse gamma process and a composed gamma process. They can be seen as 'continuous' analogues of Poisson and compound Poisson processes, respectively. For these models the first passage time distribution functions are derived. Inhomogeneous versions of these processes lead to models closely related to the Weibull failure model. All models show interesting size effects.
\end{abstract}

Keywords: Fracture theory; damage accumulation; gamma process; inverse gamma process; Weibull distribution

2000 Mathematics Subject Classification: Primary 60G51; 60K10

Secondary 60G55

\section{Introduction}

Damage accumulation models play an important role in reliability, risk, and fracture theory; see, e.g. Sobczyk (1987), (1997), Sobczyk and Spencer (1992), and Rolski et al. (1999). An important class of such models consists of those in which it is assumed that there is a point process on $[0, \infty)$ of 'events' at which damage happens. Each instance of damage is characterized by a positive damage magnitude. If the point process is a Poisson process, then the model is called a compound Poisson process (Kingman (1993)). These magnitudes accumulate until, at some time, a critical threshold is hit or exceeded. This first passage time is the quantity of interest in this paper. In the reliability context it is the lifetime of the system considered, in the risk context it characterizes the aggregate claim or total payment from a portfolio over a period, and in fracture theory it characterizes the fracture strength of a loaded sample.

In the fracture context, the points of the process represent loads at which breaks occur at flaws in the material: the flaws fail under increasing load in order of their strength. In the damage context, every failure adds some damage magnitude to an accumulating sum. Also in the physics literature, more complicated models are considered which involve the geometrical arrangement of the flaws; see, e.g. Duxbury and Leath (1994) and Todinov (2002), where clusters of flaws play a role.

The damage model sketched above shows strong discontinuity: the events happen at isolated points (with possibly large interpoint distances) and the magnitudes can be large, as shown in Figure 1. This leads to first passage time distributions which can have strange behaviours. In particular, when the threshold is small the damage accumulation process should be (nearly)

Received 23 October 2006; revision received 16 January 2007.

* Postal address: Institute of Stochastics, TU Bergakademie Freiberg, Prüferstrasse 9, D-09596 Freiberg, Germany.

** Email address: ballani@mi.fu-berlin.de 

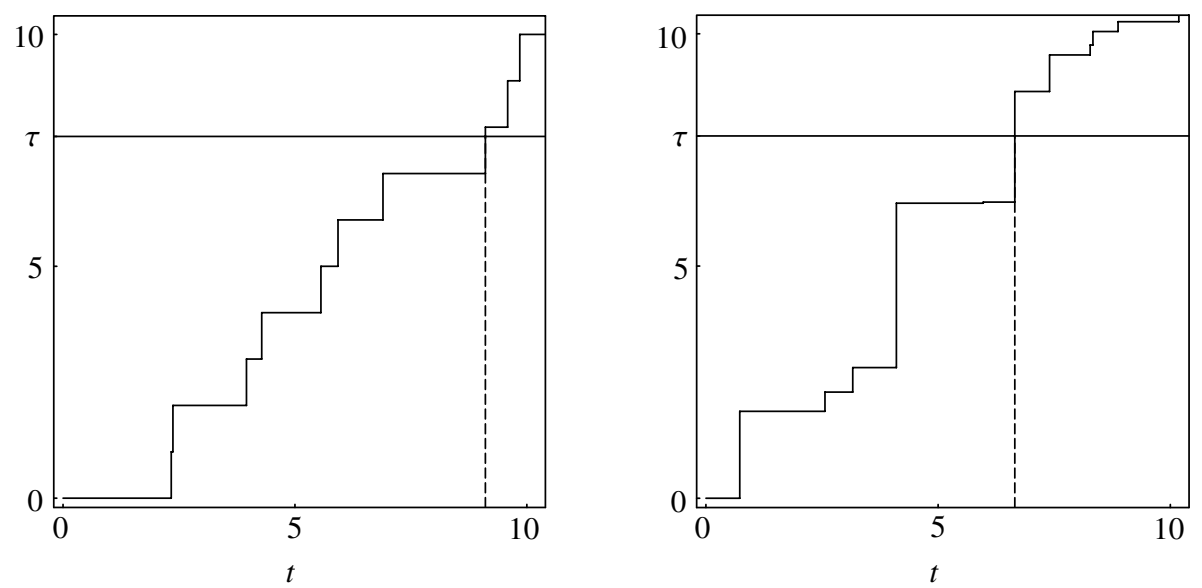

FIGURE 1: Damage accumulation processes with unit jump heights (left) and exponentially distributed jump heights (right).

continuous and nondecreasing if the results are to be reasonable. The latter property excludes Gaussian processes, which leads to inverse Gaussian distributions; see, e.g. Onar and Padgett (2000). Therefore, it may be useful to develop 'continuous' damage models in which the interpoint distances and magnitudes are 'broken up' into small pieces. This paper presents two models of such a type which are closely related to the gamma process.

A phenomenon of great interest in the fracture-theoretical interpretation of damage models is the size effect. The question is: how does the mean fracture strength, $\bar{\sigma}(V)$, of a sample depend on its size, $V$ (e.g. volume or length)? For engineers it is very important to know what happens when they go from the study of numerical simulations to that of scale models in the laboratory, and from there to full-size engineering structures. There is a large engineering literature on the size effect; see, e.g. Bažant and Planas (1998) and Krajcinovic (1996). The size effect is also of interest in the context of accelerated tests; see, e.g. Onar and Padgett (2000). The size effect can have various forms: $\bar{\sigma}(V)$ can be decreasing, increasing, or (nearly) constant in $V$. A decreasing size effect is observed in the classical Weibull model (see Section 6), but also in more complicated models; see Duxbury and Leath (1994) and Onar and Padgett (2000). It is typical in materials such as ordinary concrete. One of the models considered here has an increasing volume effect.

A nearly constant size effect is observed in materials such as ductile metals, glass ceramics (Pompe et al. (1985)), and aircrete (Wolf et al. (2005)). Pompe et al. (1985) explained the constant mean strength using a reliability model based on a network where many elements are in parallel. Also, many of the models considered in the present paper have a nearly constant mean strength and a variance of strength proportional to $1 / \mathrm{V}$.

The paper concludes with a discussion of models in which the first passage time distributions are similar to that in the classical Weibull model.

\section{Damage accumulation and first passage}

Let $\{X(t)\}$ be a stochastic process on $[0, \infty)$ which is nondecreasing and right continuous. In the following we will refer to the parameter $t$ as 'time' (in the fracture context it is 'load' or 'stress'). Let $\tau$ be a positive real number, the critical threshold. The first passage time of 
$\{X(t)\}$ over $\tau$ is

$$
T=\inf \{t: X(t) \geq \tau\} .
$$

This random variable is called the lifetime (or, in the fracture context, the fracture strength). It satisfies

$$
\mathrm{P}(T \leq t)=\mathrm{P}(X(t) \geq \tau) \text { for } t \geq 0 .
$$

In the context of the size effect studies in this paper, the threshold $\tau$ is taken to be of the form

$$
\tau=\alpha V
$$

where $\alpha$, as in Jeulin (1994), is a positive model parameter and $V$ is the sample volume; in the models below, $\{X(t)\}$ also depends on $V$ in some way. The idea behind (1) is that the sample breaks when some percentage of its flaws are broken, and the mean number of broken flaws allowed is proportional to $V$. Similar combinations of threshold and drift appear in Onar and Padgett (2000).

A popular class of models for $\{X(t)\}$ are compound processes of the form

$$
X(t)=\sum_{i=1}^{N(t)} X_{i}
$$

where $N$ is a point process on $[0, \infty)$ and $N(t)$ denotes the number of its points in $[0, t]$. The $X_{i}$ are random variables, the damage magnitudes, which can be independent and identically distributed or more general. Formulae for such processes are well known; see, e.g. Rolski et al. (1999) and Sobczyk (1997). In the present paper we use compound processes as the starting points in constructing refined models.

\section{Models of damage accumulation}

In the following, four models of damage accumulation are introduced which are both natural and promising. All four processes can play the role of $\{X(t)\}$ in Section 2.

\subsection{Poisson process}

Let $\left\{P_{\lambda}(t)\right\}$ be the homogeneous Poisson process on $[0, \infty)$ with intensity $\lambda$ as defined in Daley and Vere-Jones (2003). The accumulation process has jumps of height 1 at the points of a Poisson point process with intensity $\lambda$, so the damage accumulated up to time $t$ is a random integer with Poisson distribution with parameter $\lambda t$.

The strong discontinuity of the Poisson process may detract from its attractiveness in applications, so the following models, all of which can be seen as generalizations of the Poisson process, were developed.

\subsection{Inverse gamma process}

Let $0<b, \lambda<\infty$. The probability measure on $[0, \infty)$ with density function

$$
g_{b, \lambda}(x)=\frac{\lambda^{b}}{\Gamma(b)} x^{b-1} \mathrm{e}^{-\lambda x} \quad \text { for } x \geq 0
$$

is called the gamma distribution with shape parameter $b$ and scale parameter $\lambda$. The corresponding distribution function is denoted by $G_{b, \lambda}(x)$.

The gamma process $\left\{\gamma_{b, \lambda}(t)\right\}$ is a stochastic process on $[0, \infty)$ with $\gamma_{b, \lambda}(0)=0$ and independent, homogeneous increments such that $\gamma_{b, \lambda}(t)$ has a gamma distribution with shape 
parameter $b t$ and scale parameter $\lambda$; see Ferguson (1974), Çinlar (1980), Kingman (1993), and Applebaum (2004). (In Çinlar's notation, our $\left\{\gamma_{b, \lambda}(t)\right\}$ is written $\Gamma_{0}(b, \lambda)$.) The samples are nondecreasing and right continuous, and the countably many discontinuities are everywhere dense in $[0, \infty)$. The points

$$
t_{i}=\gamma_{b, \lambda}\left(\frac{i}{b}\right), \quad i=1,2, \ldots,
$$

form a Poisson point process with intensity $\lambda$. Gamma processes can serve as approximations of compound Poisson processes; see Dufresne et al. (1991). In the reliability context, the gamma process appears, e.g. as a model for initial damage in Park and Padgett (2005).

The inverse gamma process $\left\{\gamma_{b, \lambda}^{-1}(t)\right\}$ is defined in Ferguson and Klass (1972) by

$$
\gamma_{b, \lambda}^{-1}(t)=\inf \left\{s: \gamma_{b, \lambda}(s) \geq t\right\} \quad \text { for } t \geq 0
$$

However, in order to obtain a right-continuous process it is necessary to define $\left\{\gamma_{b, \lambda}^{-1}(t)\right\}$ by

$$
\gamma_{b, \lambda}^{-1}(t)=\inf \left\{s: \gamma_{b, \lambda}(s)>t\right\} \quad \text { for } t \geq 0
$$

Also, $\left\{\gamma_{b, \lambda}^{-1}(t)\right\}$ is nondecreasing, but in comparison with the Poisson process it only has small jumps, which follows directly from the fact that the gamma process is a Lévy process of infinite activity and thus has an infinite number of jumps on every compact interval (see Applebaum (2004)). It can be seen as a generalization of the Poisson process with intensity $\lambda$, since

$$
\gamma_{b, \lambda}^{-1}\left(t_{i}\right)=\frac{i}{b} \quad \text { for } i=1,2, \ldots
$$

i.e. the process $\left\{(1 / b) P_{\lambda}(t)\right\}$ is embedded in or coupled to $\left\{\gamma_{b, \lambda}^{-1}(t)\right\}$. Figure 2 shows a simulated sample of $\left\{\gamma_{b, \lambda}^{-1}(t)\right\}$ together with the corresponding Poisson process sample.

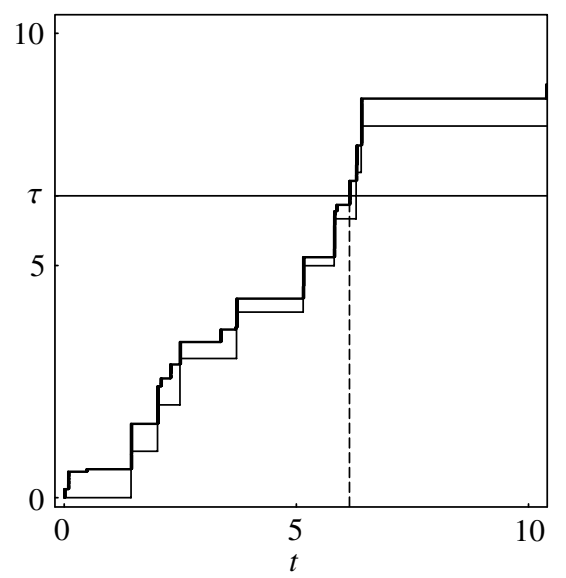

FIGURE 2: A sample of an inverse gamma process $\left\{\gamma_{b, \lambda}^{-1}(t)\right\}$ with $\lambda=b=1$ (thick line) and the corresponding embedded process $\left\{(1 / b) P_{\lambda}(t)\right\}$ (thin line). 

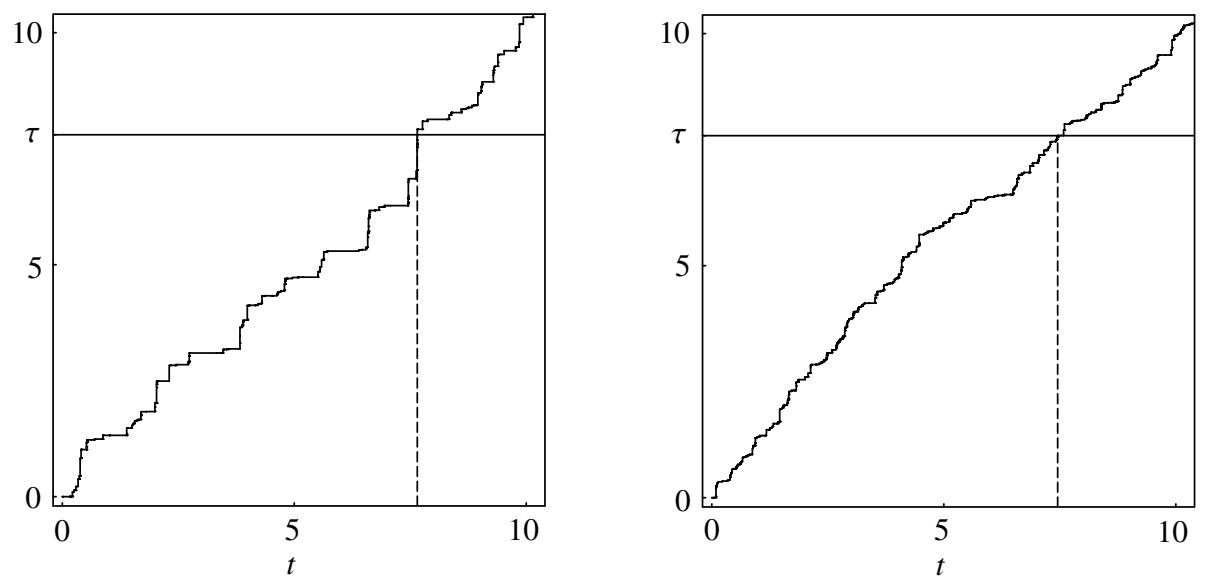

FIGURE 3: Realizations of two composed gamma processes $\left\{\gamma_{c, \kappa}\left(\gamma_{b, \lambda}^{-1}(t)\right)\right\}$ with $\lambda=b=\kappa=c=10$ (left) and $\lambda=b=\kappa=c=50$ (right).

\subsection{Gamma-Poisson process}

The gamma-Poisson process $\left\{\eta_{c, \kappa, \lambda}(t)\right\}$ can be formally defined as

$$
\eta_{c, \kappa, \lambda}(t)=\gamma_{c, \kappa}\left(P_{\lambda}(t)\right) \quad \text { for } t \geq 0,
$$

where the processes $\left\{P_{\lambda}(t)\right\}$ and $\left\{\gamma_{c, \kappa}(t)\right\}$ are as defined above and independent.

A simpler form of definition is as a compound Poisson process. Consider a homogeneous Poisson point process on $[0, \infty)$ with intensity $\lambda$ and construct a random step function,

$$
h_{i}=\sum_{j=1}^{i} e_{j},
$$

which has jumps of random heights $e_{i}$ at the Poisson process points $t_{i}$, letting the jumps be independent, identically exponentially distributed random variables with mean $\kappa^{-1}$. The corresponding stochastic process on $[0, \infty)$ is well known and used in, e.g. risk theory; see Rolski et al. (1999). It can be generalized by replacing the $e_{j}$ by gamma-distributed variates with parameters $c$ and $\kappa$, producing a gamma-Poisson process.

\subsection{Composed gamma process}

The composed gamma process $\left\{\gamma_{c, \kappa}\left(\gamma_{b, \lambda}^{-1}(t)\right)\right\}$ is constructed by composition of two independent processes, namely a gamma process with shape parameter $c$ and scale parameter $\kappa$ and an inverse gamma process defined (as above) in terms of a gamma process with shape parameter $b$ and scale parameter $\lambda$. Again, such processes are nondecreasing and right continuous, and are more variable than inverse gamma processes.

Figure 3 shows two simulated composed gamma processes with different parameters $\lambda, b$, $\kappa$, and $c$.

\section{First passage distributions and their parameters}

In this section we present formulae for the lifetime distributions of the four models in Section 3. They are well known for the first and third models, simple for the second model 
and more complicated for the fourth model. The distribution function (DF) of $T$ is denoted by $F_{\tau}(t)$ and its density function, mean, and variance by $f_{\tau}, \mu(\tau)$, and $\sigma^{2}(\tau)$, respectively.

\subsection{Poisson process}

For the Poisson process,

$$
\begin{array}{cc}
F_{\tau}(t)=1-\sum_{i=0}^{n-1} \frac{(\lambda t)^{i}}{i !} \mathrm{e}^{-\lambda t} & \text { for } t \geq 0, \\
f_{\tau}(t)=\frac{\lambda^{n} t^{n-1}}{(n-1) !} \mathrm{e}^{-\lambda t} \quad \text { for } t \geq 0, \\
\mu(\tau)=\frac{n}{\lambda}, \\
\sigma^{2}(\tau)=\frac{n}{\lambda^{2}},
\end{array}
$$

where

$$
n=\lfloor\tau\rfloor+1,
$$

$\lfloor\cdot\rfloor$ denoting the integer-part function.

\subsection{Inverse gamma process}

By construction of the inverse gamma process, $T$ has a gamma distribution with parameters $b \tau$ and $\lambda$, i.e.

$$
F_{\tau}(t)=G_{b \tau, \lambda}(t)
$$

Consequently,

$$
\begin{aligned}
\mu(\tau) & =\frac{b \tau}{\lambda}, \\
\sigma^{2}(\tau) & =\frac{b \tau}{\lambda^{2}} .
\end{aligned}
$$

\subsection{Gamma-Poisson process}

The formulae for the gamma-Poisson process with $c=1$ can be found in the literature, and the details of this are sufficient in the present paper. According to Rolski et al. (1999, p. 104) and Sobczyk (1987, p. 663),

$$
F_{\tau}(t)=1-\mathrm{e}^{-\lambda t}\left(1+\sum_{n=1}^{\infty} \frac{\lambda^{n} t^{n}}{n !(n-1) !} \gamma(n, \tau \kappa)\right),
$$

where

$$
\gamma(x, y)=\int_{0}^{y} t^{x-1} \mathrm{e}^{-t} \mathrm{~d} t
$$

denotes the (lower) incomplete gamma function (see Gautschi (1998) for its properties). For the mean and variance we have

$$
\begin{aligned}
\mu(\tau) & =\frac{1+\tau \kappa}{\lambda}, \\
\sigma^{2}(\tau) & =\frac{1+2 \tau \kappa}{\lambda^{2}} .
\end{aligned}
$$




\subsection{Composed gamma process}

Lemma 1. Let $\tau>0$ and let $m$ be a nonnegative integer. Then

$$
\lim _{x \rightarrow \infty} x^{m} \frac{\gamma(x, \tau)}{\Gamma(x)}=0 .
$$

Proof. From Gautschi (1998, Equation (5.4)), for $x>1$ it follows that

$$
0<\frac{\gamma(x, \tau)}{\Gamma(x)}<\left(1-\mathrm{e}^{-\tau}\right)^{x} .
$$

Since $0<1-\mathrm{e}^{-\tau}<1$, we have $\lim _{x \rightarrow \infty} x^{m}\left(1-\mathrm{e}^{-\tau}\right)^{x}=0$ and, hence,

$$
\lim _{x \rightarrow \infty} x^{m} \frac{\gamma(x, \tau)}{\Gamma(x)}=0 .
$$

Theorem 1. (Standardized case.) For $c, b, \kappa, \lambda=1$, the DF of $T$ is given by

$$
F_{\tau}(t)=\int_{0}^{t} \mathrm{e}^{-v} \int_{0}^{\infty} \frac{v^{x-1}}{\Gamma(x)^{2}} \int_{0}^{\tau} u^{x-1} \mathrm{e}^{-u}\left(\frac{\Gamma^{\prime}(x)}{\Gamma(x)}-\ln u\right) \mathrm{d} u \mathrm{~d} x \mathrm{~d} v,
$$

and its mean and variance are respectively

$$
\mu(\tau)=\int_{0}^{\infty} \frac{\gamma(x, \tau)}{\Gamma(x)} \mathrm{d} x
$$

and

$$
\sigma^{2}(\tau)=\int_{0}^{\infty}(2 x+1) \frac{\gamma(x, \tau)}{\Gamma(x)} \mathrm{d} x-\left(\int_{0}^{\infty} \frac{\gamma(x, \tau)}{\Gamma(x)} \mathrm{d} x\right)^{2} .
$$

Proof. Denote by $D_{\tau}(x)$ the distribution function of $\gamma_{1,1}^{-1}(\tau)$. Then

$$
\begin{aligned}
D_{\tau}(x) & =1-\mathrm{P}\left(\gamma_{1,1}^{-1}(\tau) \geq x\right) \\
& =1-\mathrm{P}\left(\gamma_{1,1}(x) \leq \tau\right) \\
& =1-G_{x, 1}(\tau) \\
& =1-\frac{1}{\Gamma(x)} \int_{0}^{\tau} u^{x-1} \mathrm{e}^{-u} \mathrm{~d} u .
\end{aligned}
$$

Hence, $D_{\tau}(x)$ has a density

$$
d_{\tau}(x)=\frac{1}{\Gamma(x)}\left(\frac{\Gamma^{\prime}(x)}{\Gamma(x)} \int_{0}^{\tau} u^{x-1} \mathrm{e}^{-u} \mathrm{~d} u-\int_{0}^{\tau} u^{x-1} \mathrm{e}^{-u} \ln u \mathrm{~d} u\right) .
$$

Let $\left\{\tilde{\gamma}_{1,1}(t)\right\}$ and $\left\{\gamma_{1,1}(t)\right\}$ be independent and identically distributed. Because $\tilde{\gamma}_{1,1}(x)$ has a gamma distribution with shape parameter $x$ and scale parameter 1, the DF of $T$ can be written as

$$
\begin{aligned}
F_{\tau}(t) & =\mathrm{P}\left(\gamma_{1,1}\left(\tilde{\gamma}_{1,1}^{-1}(t)\right) \geq \tau\right) \\
& =\mathrm{P}\left(\tilde{\gamma}_{1,1}\left(\gamma_{1,1}^{-1}(\tau)\right) \leq t\right) \\
& =\int_{0}^{\infty} \mathrm{P}\left(\tilde{\gamma}_{1,1}(x) \leq t\right) d_{\tau}(x) \mathrm{d} x \\
& =\int_{0}^{\infty} \frac{1}{\Gamma(x)} \int_{0}^{t} v^{x-1} \mathrm{e}^{-v} \mathrm{~d} v d_{\tau}(x) \mathrm{d} x
\end{aligned}
$$


Since $\tilde{\gamma}_{1,1}(x) \sim G_{x, 1}$, for all $n=1,2, \ldots$ we have

$$
\begin{aligned}
\mathrm{E}\left[\tilde{\gamma}_{1,1}(x)^{n}\right] & =\int_{0}^{\infty} z^{n} \frac{1}{\Gamma(x)} z^{x-1} \mathrm{e}^{-z} \mathrm{~d} z \\
& =\frac{1}{\Gamma(x)} \int_{0}^{\infty} z^{n+x-1} \mathrm{e}^{-z} \mathrm{~d} z \\
& =\frac{\Gamma(n+x)}{\Gamma(x)} \\
& =x(x+1) \cdots(x+n-1) \\
& =: Q_{n}(x) .
\end{aligned}
$$

With $\mathscr{D}_{\tau}(x)=1-D_{\tau}(x)$, Lemma 1 implies that $\lim _{x \rightarrow \infty} Q_{n}(x) \mathscr{D}_{\tau}(x)=0$, and it is easy to see that $Q_{n}(0) \mathscr{D}_{\tau}(0)=0$. Therefore, we obtain

$$
\begin{aligned}
\mathrm{E}\left[T^{n}\right] & =\mathrm{E}\left[\left(\tilde{\gamma}_{1,1} \circ \gamma_{1,1}^{-1}\right)^{n}(\tau)\right] \\
& =\int_{0}^{\infty} Q_{n}(x) \mathrm{d} D_{\tau}(x) \\
& =-\int_{0}^{\infty} Q_{n}(x) \mathrm{d} \mathscr{D}_{\tau}(x) \\
& =-\left[Q_{n}(x) D_{\tau}(x)\right]_{0}^{\infty}+\int_{0}^{\infty} \mathscr{D}_{\tau}(x) \mathrm{d} Q_{n}(x) \\
& =\int_{0}^{\infty} \mathscr{D}_{\tau}(x) Q_{n}^{\prime}(x) \mathrm{d} x \\
& =\int_{0}^{\infty} \frac{1}{\Gamma(x)} \int_{0}^{\tau} u^{x-1} \mathrm{e}^{-u} \mathrm{~d} u Q_{n}^{\prime}(x) \mathrm{d} x \\
& =\int_{0}^{\infty} \frac{\gamma(x, \tau)}{\Gamma(x)} Q_{n}^{\prime}(x) \mathrm{d} x .
\end{aligned}
$$

The theorem is then proved by inserting $Q_{1}^{\prime}(x)=1$ and $Q_{2}^{\prime}(x)=2 x+1$ into $\mu(\tau)=\mathrm{E}[T]$ and $\sigma^{2}(\tau)=\mathrm{E}\left[T^{2}\right]-\mathrm{E}[T]^{2}$.

Theorem 2. (Generalized case.) For general choices of $c, b, \kappa$, and $\lambda$, the DF of $T$ is given by

$$
F_{\tau}(t)=\int_{0}^{\lambda t} \mathrm{e}^{-v} \int_{0}^{\infty} \frac{v^{b x / c-1}}{\Gamma(b x / c) \Gamma(x)} \int_{0}^{\kappa \tau} u^{x-1} \mathrm{e}^{-u}\left(\frac{\Gamma^{\prime}(x)}{\Gamma(x)}-\ln u\right) \mathrm{d} u \mathrm{~d} x \mathrm{~d} v
$$

and its mean and variance are respectively

$$
\mu(\tau)=\frac{b}{\lambda c} \int_{0}^{\infty} \frac{\gamma(x, \kappa \tau)}{\Gamma(x)} \mathrm{d} x
$$

and

$$
\sigma^{2}(\tau)=\frac{b^{2}}{\lambda^{2} c^{2}}\left[\int_{0}^{\infty}\left(2 x+\frac{c}{b}\right) \frac{\gamma(x, \kappa \tau)}{\Gamma(x)} \mathrm{d} x-\left(\int_{0}^{\infty} \frac{\gamma(x, \kappa \tau)}{\Gamma(x)} \mathrm{d} x\right)^{2}\right] .
$$

Proof. Since $\left\{\gamma_{c, \kappa}(t)\right\} \sim\left\{(1 / \kappa) \gamma_{1,1}(c t)\right\}$ and $\left\{\gamma_{b, \lambda}^{-1}(t)\right\} \sim\left\{(1 / b) \tilde{\gamma}_{1,1}^{-1}(\lambda t)\right\}$, the arguments of the proof of Theorem 1 can be repeated with some minor modifications. 


\section{Size effects in the homogeneous case}

It is assumed now that $\tau=\alpha V$ and $\lambda=a V$. This corresponds to the assumption that the sample breaks if a certain percentage of its flaws break, and that the mean number of broken flaws allowed is proportional to $V$.

\subsection{Poisson process}

In the Poisson process model there is a (piecewise-)decreasing size effect; see (2) and the left-hand graph in Figure 4. Also, the variance decreases with increasing volume $V$; see (3) and the right-hand graph in Figure 4. There are discontinuities at $V=k / \alpha, k=1,2, \ldots$ Furthermore, $\mu \rightarrow \alpha / a$ and $\sigma^{2} \rightarrow 0$ as $V \rightarrow \infty$.

\subsection{Inverse gamma process}

In the inverse gamma process model there is a constant size effect, i.e. considered as a function of $V, \mu(\tau)$, given in (4), has the constant value $b \alpha / a$, and the variance (see (5)) decreases with increasing volume $V$; see Figure 5.

\subsection{Gamma-Poisson process}

In the gamma-Poisson process model there is a decreasing size effect; see (6) and the lefthand graph in Figure 6. Also, the variance decreases with increasing volume $V$; see (7) and the right-hand graph in Figure 6.
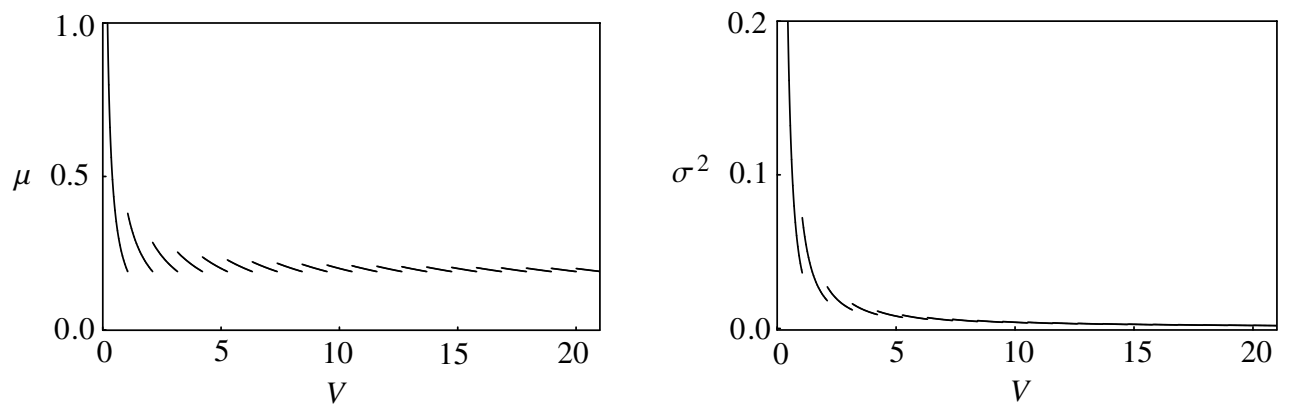

Figure 4: Mean (left) and variance (right) in the Poisson process model. The values of the parameters are $\alpha=0.95$ and $a=5$. The discontinuities result from the constant jump heights of the Poisson process.
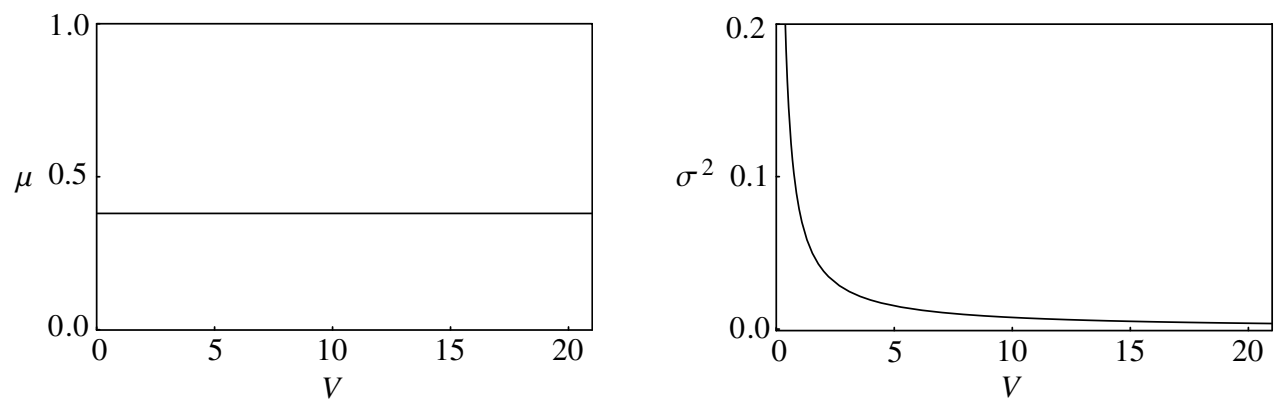

FiguRE 5: Constant mean (left) and variable variance (right) in the inverse gamma process model. The values of the parameters are $\alpha=0.95, a=5$, and $b=2$. 

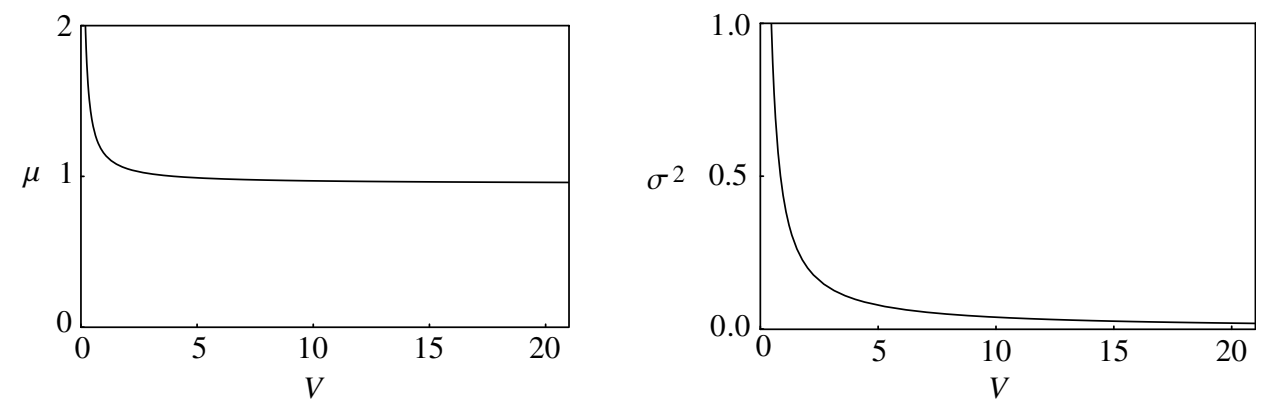

Figure 6: Mean (left) and variance (right) in the gamma-Poisson process model. The values of the parameters are $\alpha=0.95, a=5$, and $\kappa=5$.

\subsection{Composed gamma process}

Lemma 2. For $y>0$,

$$
1 \leq \frac{1}{y} \int_{0}^{\infty} \frac{\gamma(x, y)}{\Gamma(x)} \mathrm{d} x \leq 1+\frac{1}{y} .
$$

Proof. Since $\gamma(x, y) / \Gamma(x)$ is decreasing in $x>0$ and less than 1 , we have

$$
\frac{\gamma(1, y)}{0 !} \leq \frac{\gamma(x, y)}{\Gamma(x)} \leq 1, \quad x \in(0,1],
$$

and, for all $n=2,3, \ldots$,

$$
\frac{\gamma(n, y)}{(n-1) !} \leq \frac{\gamma(x, y)}{\Gamma(x)} \leq \frac{\gamma(n-1, y)}{(n-2) !}, \quad x \in(n-1, n] .
$$

Hence,

$$
\sum_{n=1}^{\infty} \frac{\gamma(n, y)}{(n-1) !} \leq \int_{0}^{\infty} \frac{\gamma(x, y)}{\Gamma(x)} \mathrm{d} x \leq \sum_{n=2}^{\infty} \frac{\gamma(n-1, y)}{(n-2) !}+1=\sum_{n=1}^{\infty} \frac{\gamma(n, y)}{(n-1) !}+1 .
$$

Since

$$
\gamma(n, y)=(n-1) !\left(1-\mathrm{e}^{-y} \sum_{k=0}^{n-1} \frac{y^{k}}{k !}\right)=(n-1) ! \mathrm{e}^{-y} \sum_{k=n}^{\infty} \frac{y^{k}}{k !}
$$

(see Gautschi (1998, Equation (1.2))), we obtain

$$
\sum_{n=1}^{\infty} \frac{\gamma(n, y)}{(n-1) !}=\mathrm{e}^{-y} \sum_{n=1}^{\infty} \sum_{k=n}^{\infty} \frac{y^{k}}{k !}=\mathrm{e}^{-y} \sum_{k=1}^{\infty} \sum_{n=1}^{k} \frac{y^{k}}{k !}=y \mathrm{e}^{-y} \sum_{k=1}^{\infty} k \frac{y^{k-1}}{k !}=y,
$$

which completes the proof of the lemma.

Lemma 3. We have

$$
\int_{0}^{\infty} x \frac{\gamma(x, y)}{\Gamma(x)} \mathrm{d} x \leq \frac{1}{2} y^{2}+2 y+1
$$


Proof. Similar to the proof of Lemma 2, we have

$$
x \frac{\gamma(x, y)}{\Gamma(x)} \leq 1, \quad x \in(0,1]
$$

and, for all $n=2,3, \ldots$,

$$
x \frac{\gamma(x, y)}{\Gamma(x)} \leq n \frac{\gamma(n-1, y)}{(n-2) !}, \quad x \in(n-1, n] .
$$

Hence,

$$
\int_{0}^{\infty} x \frac{\gamma(x, y)}{\Gamma(x)} \mathrm{d} x \leq \sum_{n=2}^{\infty} \frac{\gamma(n-1, y)}{(n-2) !} n+1=\sum_{n=1}^{\infty} \frac{\gamma(n, y)}{(n-1) !}(n+1)+1 .
$$

From (10) we have

$$
\begin{aligned}
\sum_{n=1}^{\infty} \frac{\gamma(n, y)}{(n-1) !}(n+1) & =\sum_{n=1}^{\infty} \frac{\gamma(n, y)}{(n-1) !}(n-1)+2 \sum_{n=1}^{\infty} \frac{\gamma(n, y)}{(n-1) !} \\
& =\sum_{n=1}^{\infty} \frac{\gamma(n, y)}{(n-1) !}(n-1)+2 y
\end{aligned}
$$

and from the proof of Lemma 1 we have, for $m=1$,

$$
\sum_{n=1}^{\infty} \frac{\gamma(n, y)}{(n-1) !}(n-1)=\mathrm{e}^{-y} \sum_{k=1}^{\infty} \frac{y^{k}}{k !} \sum_{n=1}^{k}(n-1)=\mathrm{e}^{-y} \sum_{k=1}^{\infty} \frac{y^{k}}{k !} \frac{1}{2} k(k-1)=\frac{y^{2}}{2} .
$$

This completes the proof of the lemma.

By substituting $\tau=\alpha V, \lambda=a V$, and $V=y / \kappa \alpha$ into (8) and (9), we obtain

$$
\mu(\tau)=\mu(y)=\frac{b \kappa \alpha}{a c} \frac{1}{y} \int_{0}^{\infty} \frac{\gamma(x, y)}{\Gamma(x)} \mathrm{d} x
$$

and

$$
\sigma^{2}(\tau)=\sigma^{2}(y)=\left(\frac{b \kappa \alpha}{a c}\right)^{2} \frac{1}{y^{2}}\left[\int_{0}^{\infty}\left(2 x+\frac{c}{b}\right) \frac{\gamma(x, y)}{\Gamma(x)} \mathrm{d} x-\left(\int_{0}^{\infty} \frac{\gamma(x, y)}{\Gamma(x)} \mathrm{d} x\right)^{2}\right] .
$$

Hence, by Lemmas 2 and 3, the composed gamma process model has a decreasing size effect with $\mu \rightarrow b \kappa \alpha / a c$ as $V \rightarrow \infty$ (see the left-hand graph in Figure 7) and

$$
\sigma^{2} \leq\left(\frac{b \kappa \alpha}{a c}\right)^{2}\left(\frac{1}{y}\left(4+\frac{c}{b}\right)+\frac{1}{y^{2}}\right)
$$

Thus, $\sigma^{2} \rightarrow 0$ as $V \rightarrow \infty$ (see the right-hand graph in Figure 7). 

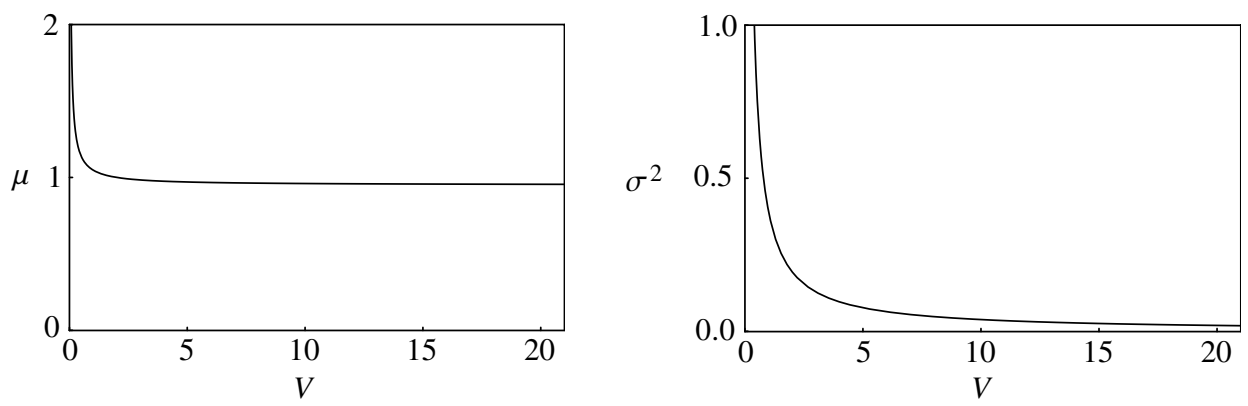

FIGURE 7: Mean (left) and variance (right) in the composed gamma process model. The values of the parameters are $\alpha=0.95, a=5, b=2, c=2$, and $\kappa=5$.

\section{Weibull-like models}

The Weibull DF results from the Weibull model, which is a good explanation for its frequent use in reliability and fracture theory. However, it depends on only two parameters, which is often not enough in applications. In the following we introduce some generalizations of the Weibull distributions which are not formal but are rather based on stochastic models.

The Weibull model can be explained as follows. In the considered sample body, of size $V$, there are flaws of different strengths. Under increasing load $t$ the flaws fail according to an inhomogeneous Poisson process with intensity function $\lambda(t)$ and points $t_{i}, 0<t_{1}<t_{2}<\cdots$. The intensity function satisfies

$$
\lambda(t)=\lambda_{0} V t^{m-1} \quad \text { for } t \geq 0,
$$

where $m$ is called the Weibull modulus. When the sample breaks at load $t$ if

$$
t_{1}<t
$$

it is said to suffer a 'weakest-link' fracture. In terms of the treatment in Section 2, we consider the case in which $\tau=1$ and $\{X(t)\}$ is an inhomogeneous Poisson process. Consequently, the DF of $T$ is

$$
F(t)=1-\exp \left\{-\left(\frac{t}{t_{0}}\right)^{m}\right\} \quad \text { for } t \geq 0,
$$

which is called the Weibull DF with modulus $m$ and $t_{0}=\left(m / \lambda_{0} V\right)^{1 / m}$.

The corresponding mean and variance are respectively

$$
\mu=\left(\frac{m}{\lambda_{0} V}\right)^{1 / m} \Gamma\left(1+\frac{1}{m}\right)
$$

and

$$
\sigma^{2}=\left(\frac{m}{\lambda_{0} V}\right)^{2 / m}\left(\Gamma\left(1+\frac{2}{m}\right)-\Gamma\left(1+\frac{1}{m}\right)^{2}\right) ;
$$

see Evans et al. (1993) and Mann et al. (1974). Equations (13) and (14) show that there is a decreasing size effect with $\sigma^{2} \rightarrow 0$ as $V \rightarrow \infty$.

The other models in Section 3 can also be generalized, either by introducing an inhomogeneity according to (11) or a time change as in Çinlar (1980). The formally simplest approach 
is to use the time transformation

$$
t \mapsto \lambda_{0} V \frac{t^{m}}{m},
$$

i.e. replace the process $\{X(t)\}$ by $\{Y(t)\}$ with

$$
Y(t)=X\left(\lambda_{0} V \frac{t^{m}}{m}\right) \quad \text { for } t \geq 0 .
$$

With $T_{\mathrm{W}}$ denoting the first passage time of $\{Y(t)\}$ over $\tau$ and $T$ denoting that of $\{X(t)\}$, (15) implies that

$$
\mathrm{P}\left(T_{\mathrm{W}} \leq t\right)=\mathrm{P}(Y(t) \geq \tau)=\mathrm{P}\left(X\left(\lambda_{0} V \frac{t^{m}}{m}\right) \geq \tau\right)=\mathrm{P}\left(T \leq \lambda_{0} V \frac{t^{m}}{m}\right) .
$$

For a weakest-link fracture, i.e. with $\tau=1$, for the various other processes this leads to the following DFs, where without loss of generality we can set $\lambda=1$.

Inverse gamma process. By (16), for the inverse gamma process we have

$$
F(t)=G_{b, 1}\left(\lambda_{0} V \frac{t^{m}}{m}\right)=\frac{1}{\Gamma(b)} \int_{0}^{\lambda_{0} V t^{m} / m} x^{b-1} \mathrm{e}^{-x} \mathrm{~d} x,
$$

where for $b=1$ the Weibull DF in (12) is obtained. Hence,

$$
\mu=\left(\frac{m}{\lambda_{0} V}\right)^{1 / m} \frac{\Gamma(b+1 / m)}{\Gamma(b)}
$$

and

$$
\sigma^{2}=\left(\frac{m}{\lambda_{0} V}\right)^{2 / m}\left(\frac{\Gamma(b+2 / m)}{\Gamma(b)}-\frac{\Gamma(b+1 / m)^{2}}{\Gamma(b)^{2}}\right) .
$$

Gamma-Poisson process. For the gamma-Poisson process, the first passage time $T_{\mathrm{W}}$ has distribution function

$$
F(t)=1-\exp \left\{-\lambda_{0} V \frac{t^{m}}{m}\right\}\left(1+\sum_{n=1}^{\infty} \frac{\left(\lambda_{0} V t^{m} / m\right)^{n}}{n !(n-1) !} \gamma(n, \kappa)\right)
$$

and its mean and variance are respectively

$$
\mu=\left(\frac{m}{\lambda_{0} V}\right)^{1 / m}\left(\Gamma\left(1+\frac{1}{m}\right)+\mathrm{e}^{-\kappa} \sum_{l=1}^{\infty} \frac{\kappa^{l}}{l !} \sum_{n=1}^{l} \frac{\Gamma(n+1 / m)}{n ! m}\right)
$$

and

$$
\begin{aligned}
\sigma^{2}=\left(\frac{m}{\lambda_{0} V}\right)^{2 / m} & \left(\Gamma\left(1+\frac{2}{m}\right)+\mathrm{e}^{-\kappa} \sum_{l=1}^{\infty} \frac{\kappa^{l}}{l !} \sum_{n=1}^{l} \frac{\Gamma(1+n+2 / m)-n \Gamma(n+2 / m)}{n !}\right. \\
& \left.-\left(\Gamma\left(1+\frac{1}{m}\right)+\mathrm{e}^{-\kappa} \sum_{l=1}^{\infty} \frac{\kappa^{l}}{l !} \sum_{n=1}^{l} \frac{\Gamma(n+1 / m)}{n ! m}\right)^{2}\right)
\end{aligned}
$$


Composed gamma process. For the composed gamma process, the first passage time $T_{\mathrm{W}}$ has distribution function

$$
F(t)=\int_{0}^{\lambda_{0} V t^{m} / m} \mathrm{e}^{-v} \int_{0}^{\infty} \frac{v^{b x / c-1}}{\Gamma(b x / c) \Gamma(x)} \int_{0}^{\kappa} u^{x-1} \mathrm{e}^{-u}\left(\frac{\Gamma^{\prime}(x)}{\Gamma(x)}-\ln u\right) \mathrm{d} u \mathrm{~d} x \mathrm{~d} v,
$$

and its mean and variance are respectively

$$
\mu=\left(\frac{m}{\lambda_{0} V}\right)^{1 / m} \int_{0}^{\infty} \frac{\gamma(c x / b, \kappa)}{\Gamma(c x / b)} \frac{\Gamma(x+1 / m)}{\Gamma(x)}\left(\frac{\Gamma^{\prime}(x+1 / m)}{\Gamma(x+1 / m)}-\frac{\Gamma^{\prime}(x)}{\Gamma(x)}\right) \mathrm{d} x
$$

and

$$
\begin{aligned}
\sigma^{2}=\left(\frac{m}{\lambda_{0} V}\right)^{2 / m} & \left(\int_{0}^{\infty} \frac{\gamma(c x / b, \kappa)}{\Gamma(c x / b)} \frac{\Gamma(x+2 / m)}{\Gamma(x)}\left(\frac{\Gamma^{\prime}(x+2 / m)}{\Gamma(x+2 / m)}-\frac{\Gamma^{\prime}(x)}{\Gamma(x)}\right) \mathrm{d} x\right. \\
& \left.-\left(\int_{0}^{\infty} \frac{\gamma(c x / b, \kappa)}{\Gamma(c x / b)} \frac{\Gamma(x+1 / m)}{\Gamma(x)}\left(\frac{\Gamma^{\prime}(x+1 / m)}{\Gamma(x+1 / m)}-\frac{\Gamma^{\prime}(x)}{\Gamma(x)}\right) \mathrm{d} x\right)^{2}\right) .
\end{aligned}
$$

Since (16) implies that

$$
\mathrm{E}\left[T_{\mathrm{W}}^{n}\right]=\mathrm{E}\left[\left(\frac{m T}{\lambda_{0} V}\right)^{n / m}\right]=\left(\frac{m}{\lambda_{0} V}\right)^{n / m} \mathrm{E}\left[T^{n / m}\right],
$$

and for a weakest-link fracture, i.e. with $\tau=1, T$ does not depend on $V$, in all Weibull-like models there is a decreasing size effect for $\mu$.

In comparison to the Weibull model, the Weibull-like models are similar but more variable since they have more parameters. In particular, when varying parameter $b$ and fixing both $\mu$ and $m$, the corresponding model based on the inverse gamma process can have a smaller variance $(b>1)$ or a larger variance $(b<1)$ than the Weibull model.

By composing the ideas of damage accumulation and inhomogeneity, we obtain another class of models which can also have increasing size effects. With $\lambda(t)$ as in (11), for $\tau=\alpha V$ the inverse gamma process model has a first passage time with distribution function

$$
F_{\tau}(t)=\frac{1}{\Gamma(b \tau)} \int_{0}^{\lambda_{0} V t^{m} / m} x^{b \tau-1} \mathrm{e}^{-x} \mathrm{~d} x
$$

mean

$$
\mu(\tau)=\left(\frac{m}{\lambda_{0} V}\right)^{1 / m} \frac{\Gamma(b \tau+1 / m)}{\Gamma(b \tau)}
$$

and variance

$$
\sigma^{2}(\tau)=\left(\frac{m}{\lambda_{0} V}\right)^{2 / m}\left(\frac{\Gamma(b \tau+2 / m)}{\Gamma(b \tau)}-\frac{\Gamma(b \tau+1 / m)^{2}}{\Gamma(b \tau)^{2}}\right) .
$$

It is easy to see that $m=1$ corresponds to the homogeneous case (see Section 4). For $m<1$ the mean first passage time is decreasing in $V$, whereas for $m>1$ there is an increasing size effect; see Figure 8. 


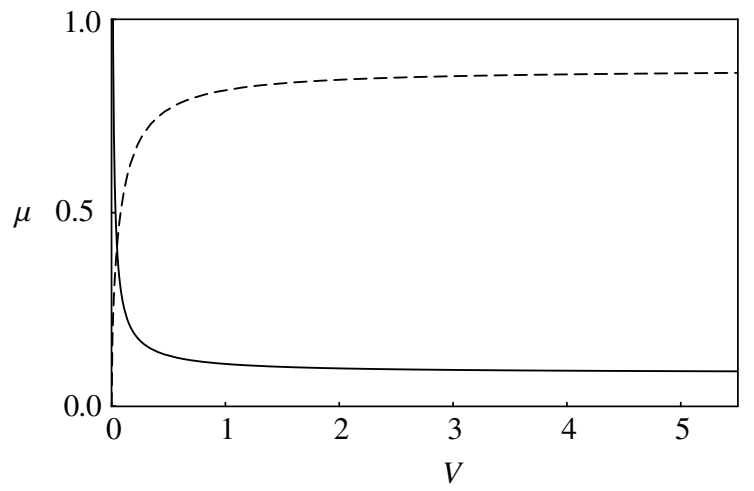

FIGURE 8: Mean in the inverse gamma process model. The values of the parameters are $\alpha=0.95, \lambda_{0}=5$, $b=2$, and $m=0.6$ (solid) and $m=2$ (dashed).

\section{Conclusion and discussion}

As the results of the paper show, plausible models can be created which have different size effects. The Weibull model, the discrete accumulation model with exponentially distributed damages, the corresponding continuous model, and a variety of Weibull-like models exhibit a decreasing size effect; the discrete accumulation model with constant damages exhibits a piecewise-decreasing size effect; its continuous generalization exhibits a constant size effect; and inhomogeneous generalizations of the latter can exhibit either increasing or decreasing size effects.

Two issues related to the fracture-theoretic application of the model considered here remain open for further research. The Poisson processes in Sections 3 and 6 result from spatial Poisson process models for the distribution of flaws in the sample. The question is: are there spatial models (perhaps of random measures) for the spatial distribution of 'flaws' which lead in a similar way to in the Poisson case to the processes considered here, in particular to the inverse gamma process? Furthermore, in some fracture-theoretic models with flaws of variable sizes (leading to variable jump heights) the flaws fail in order of size: first the large flaws and then the small ones. In such models the $e_{j}$ in the compound Poisson process would appear in decreasing order. Refining the considered models in this sense is an interesting problem.

\section{Acknowledgements}

The authors thank K. Sobczyk and D. Jeulin for stimulating discussions. They are very grateful to J. Mecke for the idea of using gamma and inverse gamma processes.

\section{References}

Applebaum, D. (2004). Lévy Processes and Stochastic Calculus. Cambridge University Press.

Bažant, Z. P. and Planas, J. (1998). Fracture and Size Effect in Concrete and other Quasibrittle Materials. CRC Press, Washington, DC.

Çınlar, E. (1980). On a generalization of gamma processes. J. Appl. Prob. 17, 467-480, 893.

Daley, D. J. And Vere-Jones, D. (2003). An Introduction to the Theory of Point Processes, Vol. 1, 2nd edn. Springer, New York.

Dufresne, F., Gerber, H. U. And Shiu, E. S. W. (1991). Risk theory with the gamma process. ASTIN Bull. 22, $177-192$. 
Duxbury, P. M. And Leath, P. L. (1994). Exactly solvable models of material breakdown. Phys. Rev. B 49, 12676-12686.

Evans, M., Hastings, N. and Peacock, B. (1993). Statistical Distributions, 2nd edn. John Wiley, New York.

Ferguson, T. S. (1974). Prior distributions of spaces of probability measures. Ann. Statist. 2, 615-629.

Ferguson, T. S. AND KLASS, M. J. (1972). A representation of independent increment processes without Gaussian components. Ann. Math. Statist. 43, 1634-1643.

Gautschi, W. (1998). The incomplete gamma functions since Tricomi. In Tricomi's Ideas and Contemporary Applied Mathematics (Atti dei Convegni Lincei 147), Accademia Nazionale dei Lincei, Roma, pp. 203-237.

JEULIN, D. (1994). Random structure models for composite media and fracture statistics. In Advances in Mathematical Modelling of Composite Materials, ed. K. Markov, World Scientific, Singapore, pp. 239-289.

Kingman, J. F. C. (1993). Poisson Processes (Oxford Studies Prob. 3). Oxford University Press.

Krajcinovic, D. (1996). Damage Mechanics, Applied Mathematics and Mechanics. Elsevier, Amsterdam.

Mann, N. R., Schafer, R. E. And SingPurwalla, N. D. (1974). Methods for Statistical Analysis of Reliability and Life Data. John Wiley, New York.

ONAR, A. AND PADGETT, W. J. (2000). Inverse Gaussian accelerated test models based on cumulative damage. J. Statist. Comput. Simul. 64, 233-247.

PARK, C. AND PAdgetT, W. J. (2005). New cumulative damage models for failure using stochastic processes as initial damage. IEEE Trans. Reliab. 54, 530-540.

Pompe, W. et al. (1985). Mechanical Properties of Brittle Materials, Modern Theories and Experimental Evidence. Elsevier, Amsterdam.

Rolski, T., Schmidli, H., Schmidt, H. and Teugels, J. (1999). Stochastic Processes for Insurance and Finance. John Wiley, New York.

SobCZYк, K. (1987). Stochastic models for fatigue damage of materials. Adv. Appl. Prob. 19, 652-673.

SoвCZYк, K. (1997). On cumulative jump models for random deterioration processes. Ann. Univ. Maria CurieSklodowska, Lublin 15, 145-157.

Sobczyk, K. And Spencer, B. F. (1992). Random Fatigue: From Data to Theory. Academic Press, Boston, MA.

Todinov, M. T. (2002). Statistics of defects in one-dimensional components. Comput. Mat. Sci. 24, 430-442.

Wolf, S., Wiegand, S., Stoyan, D. And Walther, H. B. (2005). The compressive strength of AAC - a statistical investigation. In Autoclaved Aerated Concrete. Innovation and Design, Taylor and Francis, London, pp. 287-295. 